\title{
Flow with Slip Between Coaxial Cylinders and in an Equilateral Triangular Pipe
}

\author{
John Lekner*
}

The MacDiarmid Institute for Advanced Materials and Nanotechnology, and School of Chemical and Physical Sciences, Victoria University of Wellington, P.O. Box 600, Wellington, New Zealand

\begin{abstract}
The Navier-Stokes equations are solved exactly, assuming the Navier slip boundary condition, for flow between coaxial circular cylinders (with possibly different slip lengths at the inner and outer walls), and flow in an equilateral triangular pipe. The velocity fields and total flow rates are found analytically. A relatively small slip length can lead to a large increase in the total rate of fluid flow: in the triangular pipe case the flow doubles when the slip length is only about $7.5 \%$ of the triangle side.
\end{abstract}

\section{INTRODUCTION}

The idea of slip goes back to Navier [1] in 1823. Later works by Helmholtz and Piotrowski and by Kundt and Warburg were discussed by Maxwell in 1879 [2]. The recent interest is vigorous [3-25]. Briefly, flow through nanometerscale channels has shown slip of liquid over solid when the interface is hydrophobic. The experimental data [references $3-9,11-13,21$ and 24] indicate that slip lengths of the order of 10 to $50 \mathrm{~nm}$ characterize liquid flow over hydrophobic surfaces. (Slip lengths from $200 \mathrm{~nm}$ to $20 \mu \mathrm{m}$ have been observed in nanograted superhydrophobic microchannels $[12,19]$.) Thus slip can become important (or even dominant) for very small channels.

Here we shall consider flow with slip in some pipes of simple cross-sections, assuming a uniform slip length $s$, as defined (implicitly) by Navier,

$s=\left(\frac{v_{/ l}}{\partial_{\perp} v_{/ /}}\right)_{\text {wall }}$

where $v_{/ /}$is the velocity component along the wall. The normal derivative $\partial_{\perp} v_{/ /}$is understood to be taken inward into the pipe from the wall or walls.

In general, for incompressible steady flow is in the $z$ direction, the Navier-Stokes equations reduce to $\partial_{x} p=0=\partial_{y} p$ and

$\left(\partial_{x}^{2}+\partial_{y}^{2}\right) v_{z}=\frac{1}{\eta} \partial_{z} p$

where $\eta$ is the fluid viscosity and $p$ is the pressure. For uniform flow $v_{z}$ does not depend on $z$, and so the pressure

*Address correspondence to this author at the MacDiarmid Institute for Advanced Materials and Nanotechnology, and School of Chemical and Physical Sciences, Victoria University of Wellington, P.O. Box 600, Wellington, New Zealand; E-mail: john.lekner@vuw.ac.nz gradient $\partial_{z} p$ is constant. Hence the flow problem is solved when the two-dimensional Laplacian of $v_{z}$ is constant, and (1) is satisfied.

Our new results are for flow with slip between coaxial circular cylinders, and within a pipe of equilateral triangular cross-section. For completeness we shall begin with two simple well-known solutions.

\section{Flow Between Parallel Plates}

Let the flow be in the $\mathrm{z}$ direction, between plates at $y=-h$ and $y=h$. We consider flow far away from the sides of the pipe at $x=-X$ and $+X$. When slip is absent the fluid speed is parabolic:

$v_{z}=u_{0}\left[1-\frac{y^{2}}{h^{2}}\right]$

With slip, the conditions of constant Laplacian and the slip boundary condition are also satisfied by a parabolic velocity profile:

$v_{z}=u\left[1-\frac{y^{2}}{h^{2}+2 h s}\right]$

The maximum flow speeds $u_{0}=h^{2}\left(-\partial_{z} p\right) / 2 \eta$ and $u=\left(h^{2}+2 h s\right)\left(-\partial_{z} p\right) / 2 \eta$ follow from (2).

\section{Circular Pipe}

In a circular pipe of radius $a$ the velocity (everywhere parallel to the pipe wall) is given by the usual HagenPoiseuille flow profile

$v_{z}=u_{0}\left[1-\frac{r^{2}}{a^{2}}\right]$

[the Laplacian $\left(\partial_{r}^{2}+\frac{1}{r} \partial_{r}\right) v_{z}$ is constant, and $v_{z}$ is zero at $r=a]$.

2009 Bentham Open 
With slip, the flow is again modified in a simple way:

$v_{z}=u\left[1-\frac{r^{2}}{a^{2}+2 a s}\right]$

Then $v_{/ /}=v_{z}$ and $\partial_{\perp} v_{/ /}=-\partial_{r} v_{z}$ at the wall $r=a$ are equal to $2 a u s /\left[a^{2}+2 a s\right]$ and $2 a u /\left[a^{2}+2 a s\right]$ respectively, with ratio $s$ in accord with (1). The speed $u$ determined by (2) is $u=\frac{1}{4}\left(a^{2}+2 a s\right)\left(-\partial_{z} p\right) / \eta$. The total flow rate in a circular pipe (of radius $a$, area $A=\pi a^{2}$ ) is thus

$Q=\frac{A^{2}}{8 \pi \eta}\left(-\partial_{z} p\right)\left[1+\frac{4 s}{a}\right]$

\section{CONCENTRIC CIRCULAR PIPES}

Slip-free flow between coaxial circular cylinders of radii $a$ and $b$ (with $b>a$ ) is given by

$$
v_{z}=u_{0}\left[\frac{\ln \frac{r}{a}}{\ln \frac{b}{a}}-\frac{r^{2}-a^{2}}{b^{2}-a^{2}}\right]
$$

This clearly satisfies the conditions of constant Laplacian and zero speed at $r=a$ and $r=b$. The speed $u_{0}$ determined by (2) is $u_{0}=\frac{1}{4}\left(b^{2}-a^{2}\right)\left(-\partial_{z} p\right) / \eta$. The total no-slip flow rate is

$$
Q_{0}=2 \pi \int_{a}^{b} d r r v_{z}(r)=\frac{\pi}{8 \eta}\left(-\partial_{z} p\right)\left(b^{2}-a^{2}\right)\left[a^{2}+b^{2}-\frac{b^{2}-a^{2}}{\ln (b / a)}\right]
$$

Note that $Q_{0}$ is not simply a constant times the square of the area between the pipes, $A^{2}=\pi^{2}\left(b^{2}-a^{2}\right)^{2}$, in contradistinction to $Q_{0}$ for the circular pipe (above) and the triangular pipe (next section).

Next we allow for possibly different slip lengths $s_{a}$ and $s_{b}$ at the inner and outer walls, applying (1) with the normal derivative $\partial_{\perp} v_{/ /}$taken inwardly into the pipe at both walls. The constant, $\ln (r)$ and $r^{2}$ terms which appear in the slipfree solution (8) all have constant Laplacian (zero for the constant and $\ln (r)$ terms), and can be linearly superposed in the right proportions to give the solution corresponding to slip lengths $s_{a}$ and $s_{b}$ at the inner and outer walls:

$$
\begin{aligned}
& v_{z}=u\left(\begin{array}{l}
\left(b^{2}-a^{2}\right) \ln \frac{r}{a}-\left(r^{2}-a^{2}\right) \ln \frac{b}{a}+\frac{s_{a}}{a}\left[b^{2}-r^{2}-2 a^{2} \ln \frac{b}{r}\right] \\
+\frac{s_{b}}{b}\left[2 b^{2} \ln \frac{r}{a}-\left(r^{2}-a^{2}\right)\right]
\end{array}\right. \\
& \left.+2 \frac{s_{a} s_{b}}{a b}\left(b^{2}-r^{2}\right)\right) /\left[\left(\ln \frac{b}{a}+\frac{s_{a}}{a}+\frac{s_{b}}{b}\right)\left(b^{2}-a^{2}\right)\right]
\end{aligned}
$$

The prefactor $u$ equals $u_{0}$ (this special result follows because the Laplacian of $\ln r$ is zero). At $r=a$ and $r=b$ the fluid velocity takes the values

$$
\begin{aligned}
& v_{a}=u \frac{\frac{s_{a}}{a}\left[b^{2}-a^{2}-2 a^{2} \ln \frac{b}{a}+\frac{2 s_{b}}{b}\left(b^{2}-a^{2}\right)\right]}{4\left(\ln \frac{b}{a}+\frac{s_{a}}{a}+\frac{s_{b}}{b}\right)\left(b^{2}-a^{2}\right)} \\
& v_{b}=u \frac{\frac{s_{b}}{b}\left[a^{2}-b^{2}+2 b^{2} \ln \frac{b}{a}+\frac{2 s_{a}}{a}\left(b^{2}-a^{2}\right)\right]}{4\left(\ln \frac{b}{a}+\frac{s_{a}}{a}+\frac{s_{b}}{b}\right)\left(b^{2}-a^{2}\right)}
\end{aligned}
$$

Fig. (1) shows the velocity profiles of the flow between two concentric circular cylinders, with $b=2 a$ and equal slip lengths $s_{a}=s_{b}$ varying from 0 to $0.3 a$, in steps of $0.1 a$. (For inner and outer radii of $100 \mathrm{~nm}$ and $200 \mathrm{~nm}$, the slip lengths would be 10, 20 and $30 \mathrm{~nm}$ ). The shape of the velocity profile changes very little with slip, the main change in velocity being a constant increase proportional to $s$, roughly equal to the average of $v_{a}$ and $v_{b}$. The increase also depends on $r$, but the variation with $r$ is too weak to show in the figure.

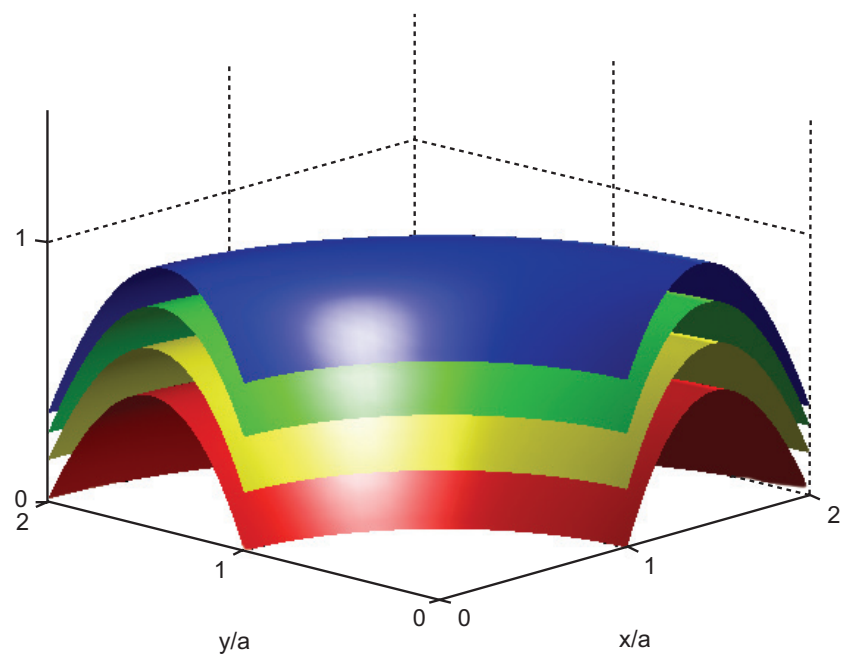

Fig. (1). Flow speed between cylindrical surfaces at $r=a$ and $r=b$, for $b=2 a$ and equal slip lengths $s_{a}=s_{b}=s$, with $s$ equal to $0,0.1 a, 0.2 a$, and $0.3 a$. The velocity profiles are shown nested, and over only one quarter of the circumference, to reveal the variation with $\mathrm{r}$.

The total flow rate when the slip lengths are $s_{a}$ and $s_{b}$ at the inner and outer cylinder walls is given by

$$
\begin{aligned}
& Q=\frac{\pi}{8 \eta}\left(-\partial_{z} p\right)\left(\begin{array}{l}
\left(b^{2}-a^{2}\right)\left[\left(a^{2}+b^{2}\right) \ln \frac{b}{a}-\left(b^{2}-a^{2}\right)\right] \\
+\frac{s_{a}}{a}\left[4 a^{4} \ln \frac{b}{a}-\left(b^{2}-a^{2}\right)\left(3 a^{2}-b^{2}\right)\right]
\end{array}\right. \\
& \begin{array}{l}
\left.+\frac{s_{b}}{b}\left[4 b^{4} \ln \frac{b}{a}-\left(b^{2}-a^{2}\right)\left(3 b^{2}-a^{2}\right)\right]\right) /\left(\ln \frac{b}{a}+\frac{s_{a}}{a}+\frac{s_{b}}{b}\right) \\
+4 \frac{s_{a} s_{b}}{a b}\left(b^{2}-a^{2}\right)^{2}
\end{array}
\end{aligned}
$$

To lowest order in $s_{a}$ and $s_{b}$, the ratio of $Q$ to $Q_{0}$ is

$$
\frac{Q}{Q_{0}} \approx 1+\frac{\frac{s_{a}}{a}\left(b^{2}-a^{2}-2 a^{2} \ln \frac{b}{a}\right)^{2}+\frac{s_{b}}{b}\left(2 b^{2} \ln \frac{b}{a}+a^{2}-b^{2}\right)^{2}}{\left(b^{2}-a^{2}\right) \ln \frac{b}{a}\left[\left(a^{2}+b^{2}\right) \ln \frac{b}{a}+a^{2}-b^{2}\right]}
$$


The flow rate increases monotonically with both $s_{a}$ and $s_{b}$. When $s_{a}=s=s_{b}$ and $b=2 a$ the total flow doubles when $s \approx 0.166 a$, so a slip length of about $17 \mathrm{~nm}$ is sufficient to double the flow between cylinders of radii $100 \mathrm{~nm}$ and $200 \mathrm{~nm}$. The total flow when $s=a$ and $b=2 a$ (corresponding to a slip length of $100 \mathrm{~nm}$ for the example above, which is well within the experimental range of slip lengths) is about 7 times the zero-slip flow, for the same pressure gradient.

We note in passing that there is no fundamental reason for the slip length to be substantially smaller than the pipe dimensions. The greatest drag reductions are obtained by modifying the surface with hydrophobic protrusions (see for example [12]), which reduce the effective contact area between the fluid and the surface. The slip lengths obtained in this way exceed $20 \mu \mathrm{m}$, for rectangular pipes in which the smallest dimension is as low as $76 \mu \mathrm{m}$ [12]. The smallest dimension can in principle be reduced further, the physical difficulty being to retain an even gap between the protrusions forming the slipping surface and the opposite wall.

The effect of slip (for given slip lengths) is clearly greatest for small inter-cylinder spacing. For small $b-a$ the leading terms in $Q_{0}$ and $Q$ are of different degree in $b-a$ :

$$
\begin{aligned}
& Q_{0}=\frac{\pi}{6 \eta}\left(-\partial_{z} p\right)\left[a(b-a)^{3}+O(b-a)^{4}\right] \\
& Q=\frac{2 \pi}{\eta}\left(-\partial_{z} p\right)\left[a(b-a)^{2} \frac{s_{a} s_{b}}{s_{a}+s_{b}}+O(b-a)^{3}\right]
\end{aligned}
$$

\section{EQUILATERAL TRIANGULAR PIPE}

So far we have considered geometries that are mathematically one-dimensional (for example, dependent only on the radial distance from the axis of symmetry). Twovariable geometries are more interesting, and more complex. One motivation for looking at a triangular geometry is curiosity about slip-flow in the corners. In the absence of slip the flow speed goes to zero linearly with the distance from the walls, except at the corners, where it goes to zero quadratically (with angular dependence). What happens with slip?

Fig. (2) shows an equilateral triangle of side $a$ with noslip laminar flow, the solution of which is well-known as one of the simplest of an infinity of solvable flows [26].

If $L=0, R=0$ and $B=0$ symbolically denote the linear equations for the left, right and basal sides of the triangle (given for a particular coordinate system in (16) below), the solution of (2) satisfying $v_{z}=0$ on the sides of the triangle is $v_{z} \sim L R B$. The value of the constant of proportionality depends on the value of the (constant) righthand side of (2), and on our choice of $L, R$ and $B$. Let the base coincide with the line $y=0$, and let the line $x=0$ bisect the triangle. Then we can take

$$
L=\frac{a}{2}+x-\frac{y}{\sqrt{3}}, \quad R=\frac{a}{2}-x-\frac{y}{\sqrt{3}}, \quad B=\frac{2 y}{\sqrt{3}}
$$

and the zero-slip velocity profile is given by

$v_{z}=\frac{27 u_{0}}{a^{3}} L R B$

(The factor $27 / a^{3}$ is chosen to make $v_{z}=u_{0}$ at the centroid of the triangle). The Laplacian of $v_{z}$ is $-36 u_{0} / a^{2}$, so (2) gives $u_{0}=\frac{a^{2}}{36 \eta}\left(-\partial_{z} p\right)$. The total rate of slip-free fluid flow through the pipe (volume per unit time) is

$Q_{0}=2 \int_{0}^{a / 2} d x \int_{0}^{\sqrt{3}\left(\frac{a}{2}-x\right)} d y v_{z}(x, y)=\frac{9 \sqrt{3}}{80} u_{0} a^{2}=\frac{A^{2}}{20 \sqrt{3} \eta}\left(-\partial_{z} p\right)$

where $A=\frac{1}{4} \sqrt{3} a^{2}$ is the area of the triangle.

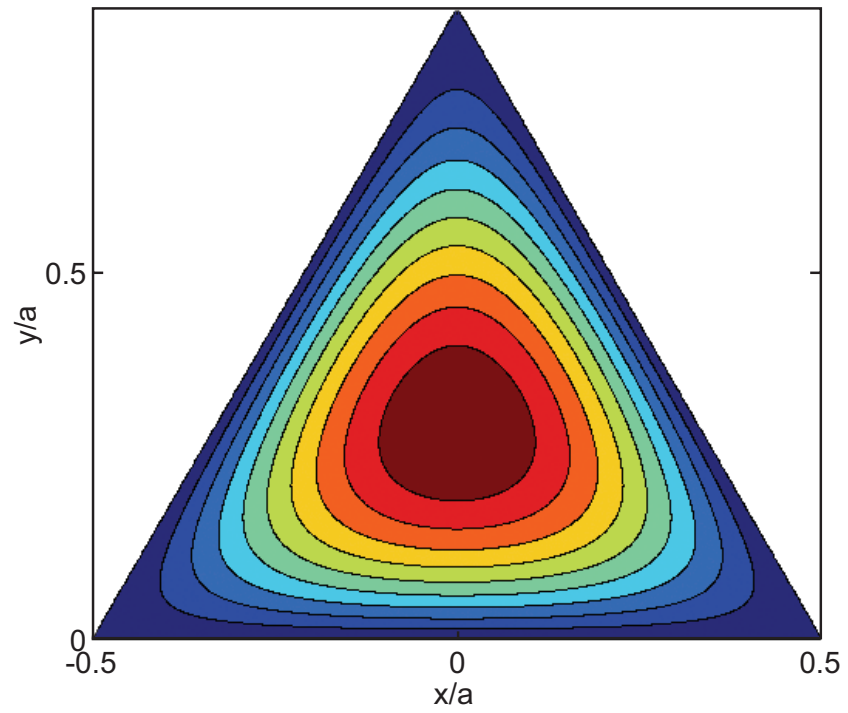

Fig. (2). Velocity distribution in an equilateral triangular pipe, without slip. The contours are drawn at 0.1 to 0.9 in increments of 0.1 of the maximum velocity (which is located at the centroid of the triangle).

Now we consider flow with slip, with constant slip length $s$. We still need to satisfy Navier-Stokes equation (2), but now the boundary condition is that $v_{z}$ on the boundary is non-zero, and equal to $s$ times its normal derivative (taken inward from the pipe walls). The symmetry of the problem, and the fact that the Laplacian of $v_{z}$ must be a constant, suggests adding terms proportional to $L R+R B+B L$ and $L+R+B$ to the zero-slip flow field (17). Note that the use of symmetry implies a particular choice of relative normalization of $L, R$ and $B$. If these are expressed as $m x+n y+c=0$, we have chosen $m^{2}+n^{2}=4 / 3$ in writing (16). With this choice we have $L+R+B=a$ and

$L R+R B+B L=\frac{a^{2}}{4}+\frac{a y}{\sqrt{3}}-x^{2}-y^{2}$ 
The trial velocity profile is taken to be

$v_{z}=\frac{27 u}{a^{3}} L R B+\frac{3 v}{a^{2}}(L R+R B+B L)+w$

The Laplacian of $v_{z}$ is a constant, namely $-(36 u+12 v) / a^{2}$, so (2) can be satisfied. To satisfy the boundary condition (1) we calculate $v_{z}$ and $\partial_{\perp} v_{z}$ on the walls. For example, on the base $B=0$ we have

$$
\begin{aligned}
& v_{z} \rightarrow \frac{3}{4} v\left[1-\left(\frac{2 x}{a}\right)^{2}\right]+w \\
& \partial_{y} v_{z} \rightarrow \frac{\sqrt{3}}{a}\left\{\frac{9}{2} u\left[1-\left(\frac{2 x}{a}\right)^{2}\right]+v\right\}
\end{aligned}
$$

The ratio of (21) to (22) will be a constant (equal to the slip length $s$ ) if

$$
v=6 \sqrt{3} u \frac{s}{a}, \quad w=18 u\left(\frac{s}{a}\right)^{2}
$$

The velocity at the centroid $\{x=0, y=a / 2 \sqrt{3}\}$ is

$V_{0}=u+v+w=u\left\{1+6 \sqrt{3} \frac{s}{a}+18\left(\frac{s}{a}\right)^{2}\right\}$

The velocity on the base $y=0$ is

$$
V_{b}=\frac{9 \sqrt{3}}{2} u \frac{s}{a}\left[1-\left(\frac{2 x}{a}\right)^{2}+\frac{4}{\sqrt{3}} \frac{s}{a}\right]
$$

At the corners $x= \pm a / 2$ this gives a quadratic dependence on the slip length:

$V_{c}=18 u\left(\frac{s}{a}\right)^{2}$

The Laplacian of $v_{z}$ is $-\frac{36 u}{a^{2}}\left(1+2 \sqrt{3} \frac{s}{a}\right)$, which equals $\left(\partial_{z} p\right) / \eta$ by (2). Thus

$$
v_{z}(x, y)=\frac{\left(-\partial_{z} p\right)}{\eta} \frac{3 L R B / a+2 \sqrt{3}(L R+R B+B L) s / a+2 s^{2}}{1+2 \sqrt{3} \frac{s}{a}}
$$

Fig. (3) shows the velocity profiles for $s$ equal to $0,0.1 a$, $0.2 a$, and $0.3 a$. Note how the flow is constrained at the vertices of the triangle, even for a substantial $s / a$ ratio.

Finally, the total flow rate is computed from (27), as in (18). We find

$$
Q=\frac{A^{2}}{20 \sqrt{3} \eta}\left(-\partial_{z} p\right)\left[1+10 \sqrt{3} \frac{s}{a}+40\left(\frac{s}{a}\right)^{2}\right] /\left[1+2 \sqrt{3} \frac{s}{a}\right]
$$

At $s=0.02 a$ the increase due to slip predicted by (28) is $27 \%$, which is remarkable for such a small slip length. The flow rate doubles when $s$ is about $7.5 \%$ of a side length; when $s=a$ the flow rate is about 13 times the zero slip value (Alternatively: at constant flow rate the pressure drop decreases by factors of 2 and 13, respectively).

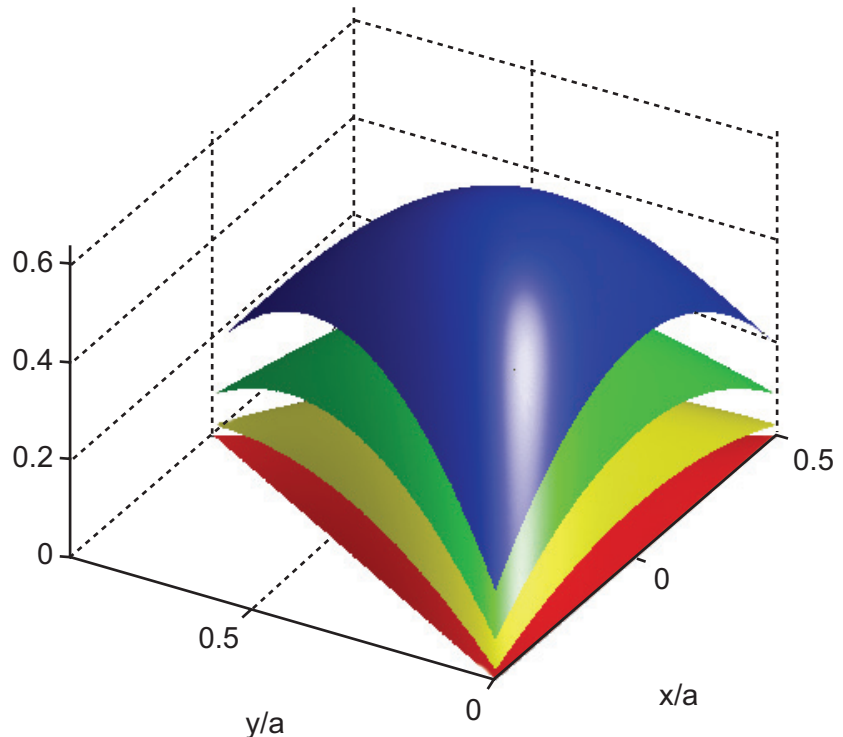

Fig. (3). Flow pattern in an equilateral triangular pipe with the slip length varying from 0 to $0.3 a$, in steps of $0.1 a$.

\section{CONCLUSION}

The Introduction gave examples of the importance of slip on fluid flow in small channels. Just how important slip is depends on the geometry as well on the relative size of the slip length to the dimensions the channels. For the circular pipe of radius $a$ and slip length $s$, the rates of flow ratio $Q / Q_{0}$ is equal to $1+4 s / a$. For the equilateral triangular pipe of side $a, Q / Q_{0}$ equals $\left(1+10 \sqrt{3} s / a+40 s^{2} / a^{2}\right) /(1+2 \sqrt{3} s / a)$. Between concentric circular cylinders of radii $a$ and $b$ the flow is more complicated (see (10) and (12)). When the slip lengths at the inner and outer walls are equal, the leading term in $Q / Q_{0}$ when $s$ is large compared to both $a$ and $b$ is

$$
\frac{4 s(b-a) \ln \frac{b}{a}}{\left(a^{2}+b^{2}\right) \ln \frac{b}{a}+a^{2}-b^{2}}
$$

For slip lengths large compared to the other pipe dimensions, the ratio of slipping flow to non-slip flow thus becomes proportional to $s$ for the circular pipe, for the concentric cylinders, and for the triangular pipe. For small slip lengths, the total flows increase linearly with slip: the leading terms are given by (13) for concentric cylinders, and by $1+8 \sqrt{3} s / a$ for the equilateral triangular pipe. However, the intermediate behaviour is quite different in the two cases.

We conclude that slip can have a dramatic effect on fluid flow when the slip lengths are commensurate with the pipe dimensions, and that the effects are strongly dependent on pipe shape.

\section{REFERENCES}

[1] Darrigol O. Worlds of flow, a history of hydrodynamics from the Bernoullis to Prandtl. Oxford: Oxford University Press 2005: pp. 117.

[2] Maxwell JC. On stresses in rarefied gases arising from inequalities of temperature. Phil Trans R Soc Lond 1879; 170: 231-56. 
[3] Pit R, Hervet H, Leger L. Direct experimental evidence of slip in hexadecane: solid interfaces. Phys Rev Lett 2000; 85: 980-3.

[4] Zhu Y, Granick S. Rate-dependent slip of Newtonian liquid at smooth surfaces. Phys Rev Lett 2001; 87: 096105.

[5] Cheng JT, Giordano N. Fluid flow through nanometer-scale channels. Phys Rev 2002; E65: 031206.

[6] Choi C-H, Westin KJA, Breuer KS. Apparent slip flows in hydrophilic and hydrophobic microchannels. Phys Fluids 2003; 15: 2897-902.

[7] Bonaccurso E, Kappl M, Butt HJ. Hydrodynamic force measurements: boundary slip of water on hydrophilic surfaces and electrokinetic effects. Phys Rev Lett 2002; 88: 076103.

[8] Zhu Y, Granick S. Limits of the hydrodynamic no-slip boundary condition. Phys Rev Lett 2002; 88: 106102.

[9] Bonaccurso E, Butt HJ, Craig VSJ. Surface roughness and hydrodynamic boundary slip of a Newtonian fluid in a completely wetting system. Phys Rev Lett 2003; 90: 144501.

[10] Lauga E, Brenner MP. Dynamic mechanisms for apparent slip on hydrophobic surfaces. Phys Rev E 2004; 70: 036311.

[11] Zhang J, Kwok D. Apparent slip over a solid-liquid interface with a no-slip boundary condition. Phys Rev E 2004; 70: 056701.

[12] Ou J, Perot B, Rothstein JP. Laminar drag reduction in microchannels using ultrahydrophobic surfaces. Phys Fluids 2004; 16: 4635-43.

[13] Cottin-Bizonne C, Cross B, Steinberger A, Charlaix E. Boundary slip on smooth hydrophobic surfaces: intrinsic effects and possible artifacts. Phys Rev Lett 2005; 94: 056102.

[14] Joseph P, Tabeling P. Direct measurement of the apparent slip length. Phys Rev E 2005; 71: 035303R.
[15] Lauga E, Cossu C. A note on the stability of slip channel flows, Phys Fluids 2005; 17: 088106.

[16] Hendy SC, Jasperse M, Burnell J. Effect of patterned slip on microand nanofluidic flows. Phys Rev E 2005; 72: 016303.

[17] Vinogradova OI, Yakubov GE. Surface roughness and hydrodynamic boundary conditions. Phys Rev E 2006; 73: 045302R.

[18] Tophoj L, Moller S, Brons M. Streamline patterns and their bifurcations near a wall with Navier slip boundary conditions. Phys Fluids 2006; 18: 083102.

[19] Choi C-H, Ulmanella U, Kim J, Ho C-M, Kim C-J. Effective slip and friction reduction in nanograted superhydrophobic microchannels. Phys Fluids 2006; 18: 087105.

[20] Priezjev NV. Rate-dependent slip boundary conditions for simple fluids. Phys Rev E 2007; 75: 051605.

[21] Huang P, Breuer KS. Direct measurement of slip length in electrolyte solutions, Phys Fluids 2007; 19: 028104

[22] Sbragaglia M, Prosperetti A. A note on the effective slip properties for microchannel flows with ultrahydrophobic surfaces. Phys Fluids 2007; 19: 043603.

[23] Hendy SC, Lund NJ. Effective slip boundary conditions for flows over nanoscale chemical heterogeneities. Phys Rev E 2007; 76: 066313.

[24] Willmott GR, Tallon JL. Measurement of Newtonian fluid slip using a torsional ultrasonic oscillator. Phys Rev E 2007; 76: 066306.

[25] Willmott GR. Dynamics of a sphere with inhomogeneous slip boundary conditions in Stokes flow. Phys Rev E 2008; 77: 055302.

[26] Lekner J. Viscous flow through pipes of various cross-sections. Eur J Phys 2007; 28: 521-7.

(C) John Lekner; Licensee Bentham Open.

This is an open access article licensed under the terms of the Creative Commons Attribution Non-Commercial License (http://creativecommons.org/licenses/by$\mathrm{nc} / 3.0 /$ ) which permits unrestricted, non-commercial use, distribution and reproduction in any medium, provided the work is properly cited. 